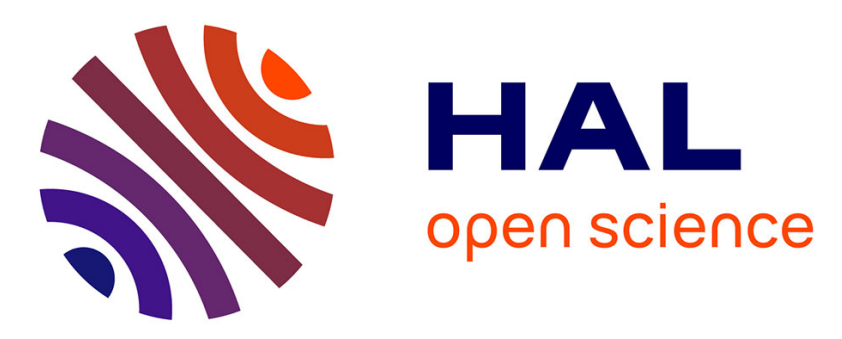

\title{
Mechanistic Investigations of Green mEos4b Reveal a Dynamic Long-Lived Dark State
}

Elke de Zitter, Jacqueline Ridard, Daniel Thédié, Virgile Adam, Bernard C. Levy, Martin Byrdin, Guillaume Gotthard, Luc van Meervelt, Peter Dedecker, Isabelle Demachy, et al.

\section{To cite this version:}

Elke de Zitter, Jacqueline Ridard, Daniel Thédié, Virgile Adam, Bernard C. Levy, et al.. Mechanistic Investigations of Green mEos4b Reveal a Dynamic Long-Lived Dark State. Journal of the American Chemical Society, 2020, 142 (25), pp.10978-10988. 10.1021/jacs.0c01880 . hal-02895781

\section{HAL Id: hal-02895781 \\ https: / hal.univ-grenoble-alpes.fr/hal-02895781}

Submitted on 20 Nov 2020

HAL is a multi-disciplinary open access archive for the deposit and dissemination of scientific research documents, whether they are published or not. The documents may come from teaching and research institutions in France or abroad, or from public or private research centers.
L'archive ouverte pluridisciplinaire HAL, est destinée au dépôt et à la diffusion de documents scientifiques de niveau recherche, publiés ou non, émanant des établissements d'enseignement et de recherche français ou étrangers, des laboratoires publics ou privés. 


\section{Mechanistic Investigations of Green mEos4b Reveal a Dynamic Long-Lived Dark State}

Elke De Zitter, Jacqueline Ridard, ${ }^{\perp}$ Daniel Thédié, ${ }^{\perp}$ Virgile Adam, Bernard Lévy, Martin Byrdin, Guillaume Gotthard, Luc Van Meervelt, Peter Dedecker, Isabelle Demachy, and Dominique Bourgeois*

Cite This: J. Am. Chem. Soc. 2020, 142, 10978-10988

Read Online

ACCESS | Llll Metrics \& More | 国 Article Recommendations | (1) Supporting Information

ABSTRACT: Green-to-red photoconvertible fluorescent proteins (PCFPs) are key players in advanced microscopy schemes such as photoactivated localization microscopy (PALM). Whereas photoconversion and red-state blinking in PCFPs have been studied intensively, their green-state photophysical behavior has received less attention. Yet dark states in green PCFPs can become strongly populated in PALM schemes and exert an indirect but considerable
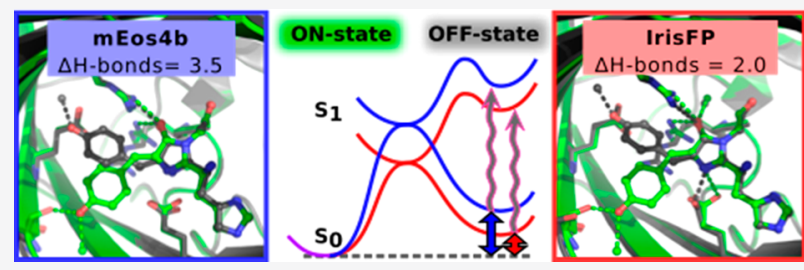
influence on the quality of data recorded in the red channel

Furthermore, green-state photoswitching in PCFPs can be used directly for PALM and has been engineered to design highly efficient reversibly switchable fluorescent proteins (RSFPs) amenable to various nanoscopy schemes. Here, we demonstrate that green $\mathrm{mEos} 4 \mathrm{~b}$ efficiently switches to a long-lived dark state through cis-trans isomerization of its chromophore, as do most RSFPs. However, by combining kinetic crystallography, molecular dynamics simulations, and Raman spectroscopy, we find that the dark state in green mEos $4 \mathrm{~b}$ is much more dynamic than that seen in switched-off green IrisFP, a biphotochromic PCFP engineered from the common EosFP parent. Our data suggest that H-bonding patterns maintained by the chromophore in green PCFPs and RSFPs in both their on- and off-states collectively control photoswitching quantum yields. The reduced number of $\mathrm{H}$-bonds maintained by the dynamic dark chromophore in green mEos $4 b$ thus largely accounts for the observed lower switching contrast as compared to that of IrisFP. We also compare the long-lived dark states reached from green and red mEos $4 \mathrm{~b}$, on the basis of their X-ray structures and Raman signatures. Altogether, these data provide a unifying picture of the complex photophysics of PCFPs and RSFPs.

\section{INTRODUCTION}

Fluorescent proteins that can change their fluorescent state upon illumination, often referred to as phototransformable fluorescent proteins (PTFPs), have played a central role in the recent development of super-resolution microscopy. ${ }^{1}$ Among those, green-to-red photoconvertible fluorescent proteins (PCFPs) irreversibly shift their emission spectra upon illumination with UV light, as a consequence of a photoinduced backbone breakage (so-called photo-Kolbe) reaction that extends the electron conjugated system of the chromophore. ${ }^{2}$ PCFPs have become highly popular markers for single-molecule localization microscopy (SMLM) ${ }^{3}$ notably in live cells and for dynamic studies based on techniques such as single-particle tracking PALM (sptPALM). ${ }^{4,5}$ On the other hand, reversibly switchable fluorescent proteins (RSFPs) are able to undergo several cycles of switching between a fluorescent on-state and a nonfluorescent off-state, most often as a consequence of light-induced cis-trans isomerization of their chromophore. ${ }^{6}$ RSFPs have found more applications in the development of super-resolution techniques such as RESOLFT (reversible saturable optical fluorescence transitions), ${ }^{7}$ NL-SIM (nonlinear structured illumination), ${ }^{8}$ or SOFI (superresolution optical fluorescence imaging), ${ }^{9,10}$ and some RSFPs have also been successfully employed in multicolor
PALM. ${ }^{11}$ Whereas PCFPs have been discovered exclusively in anthozoan species, ${ }^{12}$ RSFPs have been evolved both from hydrozoan ${ }^{13,14}$ and from anthozoan species. ${ }^{15}$ In fact, whereas green-to-red photoconversion based on the photo-Kolbe mechanism is a specific property of a limited set of fluorescent proteins that all incorporate a histidine at the first position of their chromophore triad, ${ }^{1}$ reversible photoswitching is a much more widespread characteristic exhibited by many FPs. Nevertheless, some of the most popular RSFPs of anthozoan origin such as Dronpa variants (Pectiniidae sp.) display chromophore pockets highly similar to those of well-known PCFPs such as those derived from EosFP (Lobophyllia sp.) or Dendra (Dendronephthya sp.). ${ }^{6}$ Overall, the photophysical mechanisms of PCFPs and RSFPs are highly intertwined and need to be apprehended in a global manner.

As PCFP markers are mostly used in SMLM approaches, their complex photophysical behavior has often been studied in

Received: February 17, 2020

Published: May 28, 2020 

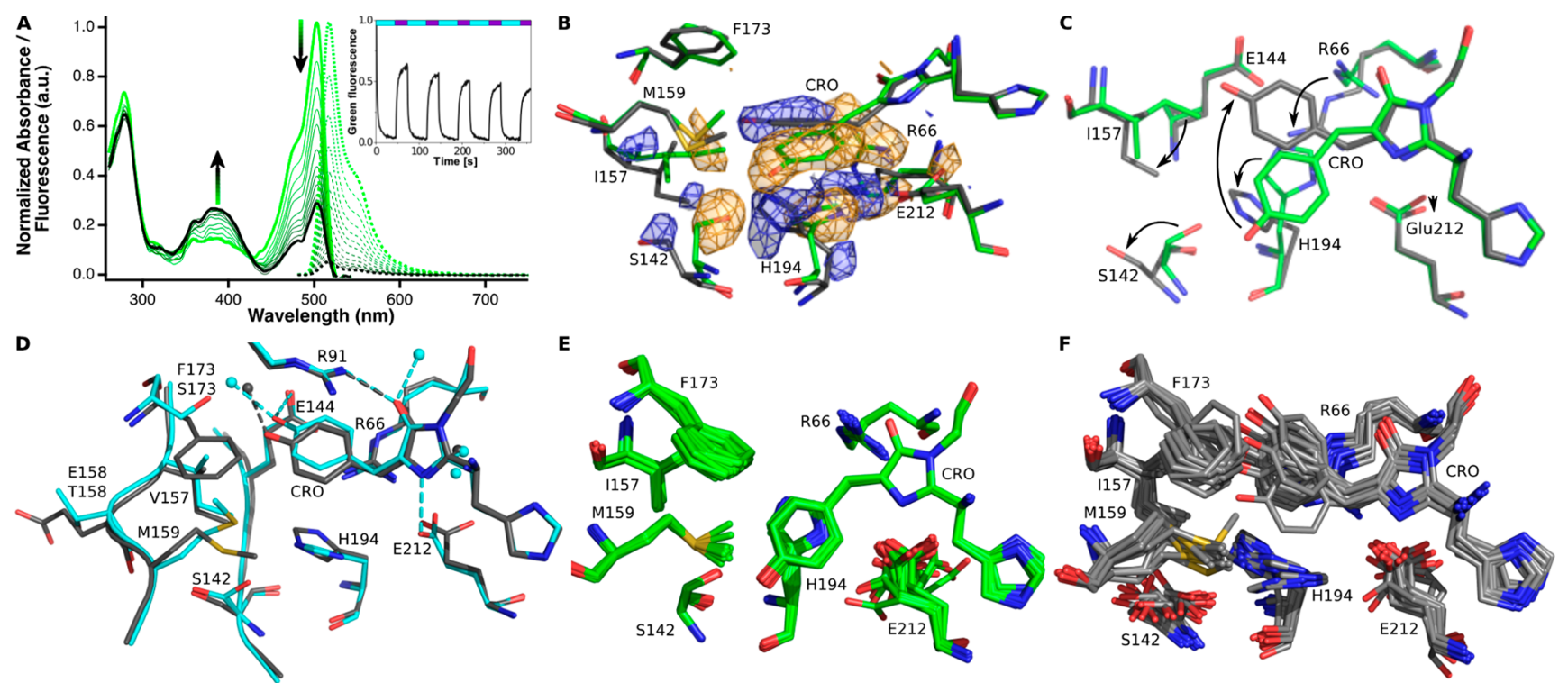

Figure 1. Long-lived dark state in green mEos4b. (A) Absorption (plain lines) and fluorescence emission (dashed lines) spectra on PVA-embedded mEos4b during $488 \mathrm{~nm}$ illumination (from green to black). Absorbance spectra are cut at $543 \mathrm{~nm}$ for clarity. See materials and methods in the Supporting Information for the higher apparent residual absorbance than fluorescence emission at the end of the illumination. The inset shows the evolution of the fluorescence during five switching cycles of subsequent cyan and UV illumination. (B) Comparison between mEos $4 \mathrm{~b}$ in its green state (green) and Green off dark state (gray) in the $F_{\text {obs,dark }}-F_{\text {calc,bright }}$ difference electron density map contoured at -3 rmsd (orange) and +3 rmsd (blue). (C) Structure of the bright and Green ${ }_{\text {off }}$ state (green and gray, respectively). Major conformational changes upon entering Green off are indicated by arrows. (D) Structural comparison between off-switched green mEos4b (Green ${ }_{\text {off }}$, gray) and off-switched green IrisFP (cyan, PDB ID: 2VVI, chain C). H-bonds possibly established between the chromophore and the surrounding pocket are indicated by dashed lines ( $\mathrm{H}-\mathrm{bond}$ criterium: distance smaller than or equal to $3.2 \AA$ ). Spheres indicate water molecules. (E) Superposition of models derived from ensemble refinement of mEos $4 \mathrm{~b}$ in its green bright state. (F) Superposition of models from ensemble refinement of mEos $4 \mathrm{~b}$ in its green dark state.

the red state and addressed at the single-molecule level. In particular, the propensity of photoconverted PCFPs to repeatedly enter reversible dark states, referred to as blinking, is of major concern, due to severe complications introduced in the interpretation of PALM data. ${ }^{16}$ Blinking in PCFPs can be categorized into three types. Fast blinking occurs on the microsecond to millisecond time scale and can be associated with transient protonation of the hydroxyl group in the para position of the hydroxybenzylidene moiety of the chromophore or to intersystem crossing to the triplet state $T_{1}$ followed by return to the ground state. ${ }^{17-20}$ Medium-rate blinking has been proposed to result from redox chemistry occurring in $T_{1}$, leading to the build-up of radical states with lifetimes in the several milliseconds time range. ${ }^{21-24}$ Recently, slow blinking has been shown to involve photoswitching through cis-trans isomerization coupled to protonation of the chromophore as observed in RSFPs. ${ }^{25}$ The associated dark times are illumination and environment sensitive $\mathrm{e}^{25-28}$ and, under typical illumination intensities used in PALM, may last hundreds of milliseconds to several tens of seconds.

The mechanisms driving green-to-red photoconversion in PCFPs have been the subject of many experimental and theoretical studies. ${ }^{29}$ However, because only the red-state fluorescence is recorded in PALM experiments, little attention has generally been given to the study of dark-state formation prior to photoconversion in these markers. Yet, recent studies have shown that green-state photophysics greatly matters. Notably, reversible photoswitching and photobleaching in that state reduce the population of fluorophores amenable to photoconversion to the red state and modify photoconversion kinetics. $^{30-32}$ Photoswitching and photobleaching of green mEos variants were shown to occur upon $488 \mathrm{~nm}$ illumination but also under strong $561 \mathrm{~nm}$ illumination, due to the so-called Urbach tail effect, which implies that the readout laser used in PALM directly affects PCFPs in their green state ${ }^{30}$ and contributes to reducing the overall photoconversion efficiency. $^{33,34}$ Whenever $488 \mathrm{~nm}$ light is used in SMLM experiments, in multicolor mode, ${ }^{35-37}$ or, as we recently suggested, to reduce long-lived blinking in sptPALM, ${ }^{25}$ greenstate photophysics is even more at play. Recently, it was proposed that combined illumination at 505 and $400 \mathrm{~nm}$ achieved faster and more efficient photoconversion of LEA, an engineered ancestral PCFP, with photoconversion arising directly from the switched-off green state. ${ }^{32}$ Interestingly, it was also demonstrated that illumination at $470 \mathrm{~nm}$ prior to photoconversion of SAASoti, a PCFP exhibiting pronounced green-state photoswitching, resulted in modified red-state photophysics after photoconversion. ${ }^{31}$

Beyond its effect on photoconversion and subsequent redstate photophysics, the engineering of reversible photoswitching in green PCFPs has also been of rising interest. The proteins IrisFP, ${ }^{38}$ mIrisFP, ${ }^{39}$ and $\mathrm{NijiFP}^{40}$ (derived from EosFP, mEosFP, and Dendra2, respectively) were engineered to exhibit rapid and efficient photoswitching in both their green- and their red-states, which enabled pulse chase twocolor SMLM experiments. ${ }^{39}$ Conversely, pcDronpa was designed to incorporate photoconversion into the RSFP Dronpa. ${ }^{41}$ Lately, green RSFPs have been engineered from PCFPs, essentially by removing the photoconversion capability of their parent proteins: this was achieved in the mGeos family (evolved from mEos2), ${ }^{42}$ in Skylan-S ${ }^{9}$ and Skylan-NS ${ }^{8}$ (evolved from mMaple3), and in G-MarsQ variants ${ }^{43}$ (evolved from mEos3.1). Finally, mEosEM, a variant of mEos $4 b$ recently engineered to exhibit strong resistance to osmium 
staining and Epon embedding, was shown to allow PALM imaging through green-state photoswitching, as green-to-red photoconversion was lost upon fixation. ${ }^{44}$

Importantly, in all nanoscopy applications relying on reversible photoswitching of the fluorescent markers, a key performance parameter is the so-called switching contrast, which largely dictates the reachable resolution gain. ${ }^{45}$ At the ensemble level, switching contrast is typically derived from the measurement of the residual steady-state fluorescence after offswitching of an RSFP. It is mostly a consequence of the propensity of the off-state chromophore to be switched back to the on-state by the employed off-switching actinic light. It is therefore important to evaluate mechanisms controlling switching contrast in the above-mentioned RSFPs.

Although several X-ray structures of RSFPs in their off-state have been published, ${ }^{10,38,41,45-48}$ the exact nature of the photoswitched state in green PCFPs that have not been engineered to exhibit strong photochromism has remained unknown to date. This is notably the case for the popular mEos variants from which most PCFP-derived RSFPs have been engineered, although the structure of a so-called "frustrated" isomerized state has been recently captured by us in photoconverted red mEos $4 \mathrm{~b} .^{25}$ mEos $4 \mathrm{~b}$ is a bright and highly monomeric variant of mEos 2 that was designed to withstand a high concentration of osmium tetroxide $\left(\mathrm{OsO}_{4}\right)$ in correlative light and electron microscopy (CLEM). ${ }^{49}$ Here, we investigate the structural basis for green-state photoswitching in mEos $4 \mathrm{~b}$, and, using molecular dynamics simulations, we compare the off-state dynamics of this PCFP with that of green IrisFP, which was designed to display efficient photoswitching and only differ from all mEos variants, at the level of the chromophore pocket, by the single mutation Phe173Ser. ${ }^{38}$ The highly dynamic chromophore and low number of $\mathrm{H}$-bonds anchoring the green chromophore to the surrounding $\beta$-barrel scaffold observed in the dark state of mEos $4 \mathrm{~b}$ provide insight into a possible mechanism controlling switching contrast in RSFPs. Finally, we present Raman spectra of green and red $\mathrm{mEos} 4 \mathrm{~b}$ in both their bright and dark states and provide a structural comparison between those latter states.

\section{RESULTS}

Photoswitching of Green mEos4b. At the ensemble level, $\mathrm{mEos} 4 \mathrm{~b}$ in its green state exhibits substantial photochromism, similar to that of mEos3.2 and slightly more pronounced than that of $\mathrm{mEos} 2^{30}$ (Figure 1A, inset, and Figure S1). Dark-state formation was characterized by the decay of the absorbance band characteristic of the anionic chromophore, which peaked at $504 \mathrm{~nm}$, and the rise of the band assigned to a protonated chromophore, which peaked at $\sim 390$ $\mathrm{nm}$ (Figure 1A). The stability of the anionic peak position and width during illumination suggests that no dark anionic species is formed (Figure S2). A comparison of the switching kinetics of green mEos $4 b$ with IrisFP, Dronpa, and mGeosM, three representative RSFPs exhibiting fast, medium, and slow photoswitching, respectively, is presented in Figure S1B. Upon illumination with $488 \mathrm{~nm}$ light, green mEos $4 \mathrm{~b}$ switches approximately 60 times slower than does green IrisFP, 5 times slower than Dronpa, and as fast as mGeosM (Figure S1B and Table S1). Back switching to the on-state of mEos $4 b$ under $405 \mathrm{~nm}$ light is however only $\sim 5$ times slower than that for IrisFP, and $\sim 1.5$ times slower than that for Dronpa. The mEos $4 \mathrm{~b}$ photoswitching contrast, calculated as the ratio between the fluorescence intensities before and after photo- switching, was measured to be $34 \pm 3$, significantly lower than the switching contrast of IrisFP $(82 \pm 19)$ but similar to those of Dronpa and mGeosM (Table S1). Thermal recovery was also measured to be slower in green mEos $4 \mathrm{~b}$ (half-time of $\sim 2.8$ $\mathrm{h})$ as compared to IrisFP $(\sim 0.5 \mathrm{~h})$ (Figure S3). As was observed previously for mEos $2,{ }^{30}$ the minimal kinetic model allowing satisfactory fit of the mEos $4 \mathrm{~b}$ on-to-off switching curve involves the formation of two reversible and one nonreversible dark states (Figure S4). The nonreversible loss of green fluorescence is mainly assigned to photobleaching, possibly through sulfoxidation of Met159 that results in a permanently protonated chromophore, ${ }^{50}$ although residual green-to-red photoconversion by $488 \mathrm{~nm}$ light may also slightly contribute. ${ }^{25,30}$ The shorter-lived reversible dark state is sensitive to light, relaxes with a rate of $\sim 0.32 \mathrm{~s}^{-1}$ at $7 \mathrm{~W} / \mathrm{cm}^{2}$ of $488 \mathrm{~nm}$ illumination (Figure S5), and therefore accumulates only transiently (Figure S4). The triplet state $\mathrm{T}_{1}$ and a shortlived dark state derived from $\mathrm{T}_{1}$ and previously assigned to a nonabsorbing radical state exhibiting a distorted chromophore $^{23}$ are likely to relax too rapidly to be detected under the low illumination intensity conditions employed here. In the following, we turned our attention to the long-lived dark state (referred to as Green $_{\text {off }}$ ) mostly responsible for the observed off-switching of mEos $4 \mathrm{~b}$.

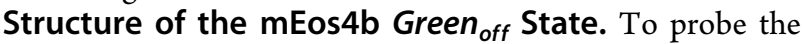
mechanism of green mEos $4 b$ associated with the formation of the long-lived dark state, we used kinetic crystallography. ${ }^{47}$ We first grew crystals and investigated their switching behavior with microspectrophotometry. ${ }^{51}$ Upon gentle illumination with $488 \mathrm{~nm}$ light, absorbance and fluorescence emission spectra recorded in crystallo (Figure S6) showed changes similar to those recorded in solution (Figure 1A). Dark-state formation was also reversible in the crystalline state, as the spectral data corresponding to the fluorescence state could be swiftly recovered by subsequent illumination with $405 \mathrm{~nm}$ light (Figure S7A).

We then selected a high-quality green mEos $4 \mathrm{~b}$ crystal, switched it under spectroscopic monitoring, cryocooled it for crystallographic data collection, and compared the obtained structure with that of mEos $4 \mathrm{~b}$ in the green fluorescent state. ${ }^{25}$ Electron difference density maps at $2.4 \AA$ resolution revealed pronounced conformational changes, indicative of cis-trans isomerization of the chromophore (Figure 1B). Structural changes were observed only in the vicinity of the chromophore pocket (Figure S8). Consistent with the predicted low occupation of all of the other dark or fluorescent states after $488 \mathrm{~nm}$ illumination (Figure S9), structural refinement of the mEos $4 \mathrm{~b}$ Green $_{\text {off }}$ state revealed an essentially fully occupied trans configuration of the chromophore. Control experiments also revealed that the observed electron difference density features disappeared upon further illumination of the crystal with gentle $405 \mathrm{~nm}$ light (Figure S7).

The structural changes that accompany dark-state formation in green mEos $4 b$ are largely similar to those seen in green anthozoan RSFPs such as Dronpa, ${ }^{52} \mathrm{pcDronpa}^{41}{ }^{41} \mathrm{IrisP}^{38}$ and mTFP0.7, ${ }^{48}$ which suggests that, despite restricted space to accommodate a trans chromophore, a similar photoswitching mechanism takes place (Figure 1C,D). Notably, the tightly $\mathrm{H}$ bonded triad Glu144-His194-Glu212 in the cis configuration is replaced by the Glu144-Arg66-Glu212 triad in the trans configuration, with His194 or Arg66 stabilizing the chromophore by $\pi$-stacking and $\pi$-cation interactions with the hydroxybenzylidene moiety, respectively (Figure S10). The 

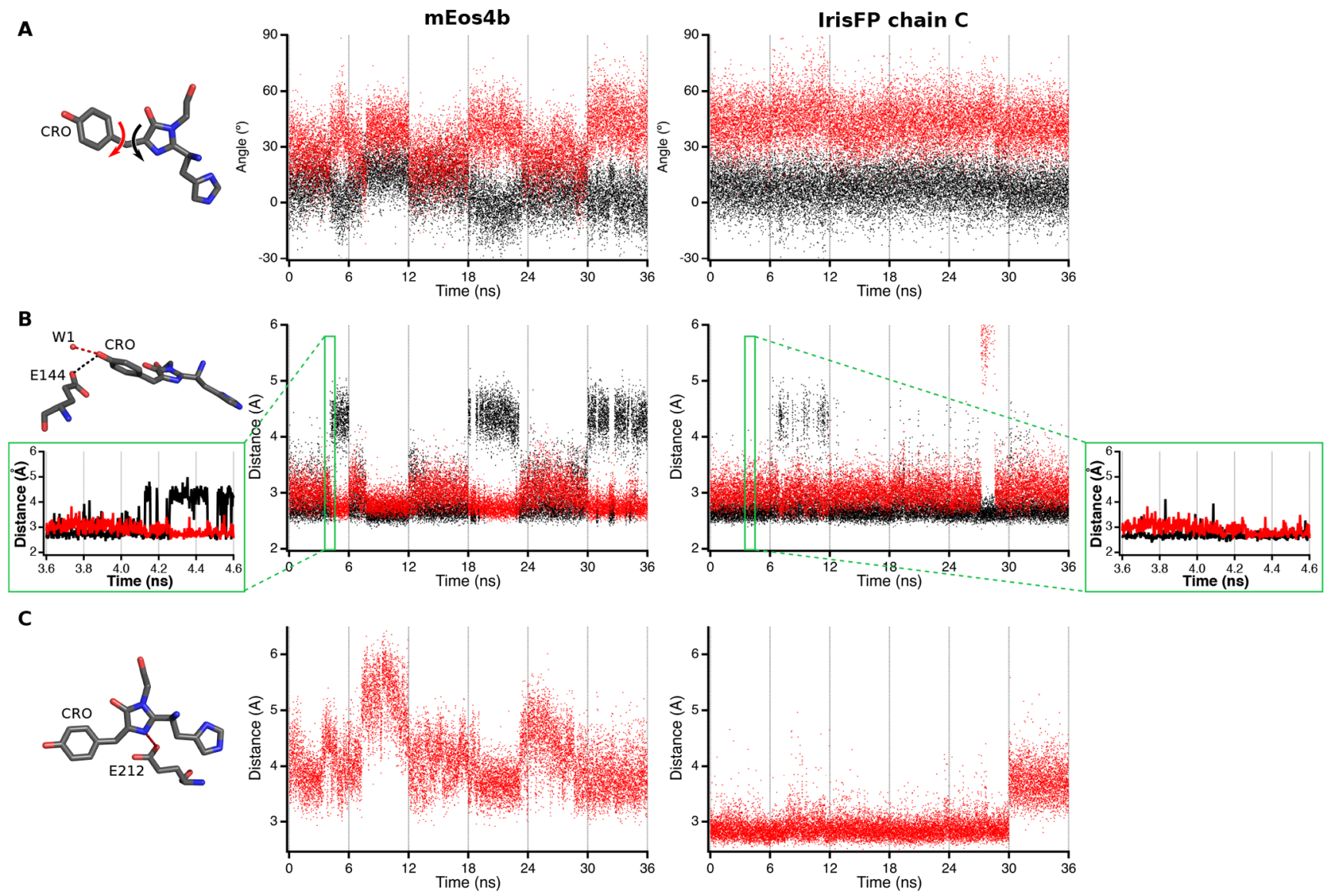

Figure 2. Comparative molecular dynamics simulation of green mEos $4 b$ and IrisFP in their switched-off state. (A) Time evolution of tilt (black) and twist (red) dihedral angles during six concatenated MD simulations (6 ns each) of mEos4b (left) and IrisFP chain C with inward Ser142 orientation (right). (B) Time evolution of the distance between the chromophore hydroxybenzylidene oxygen (O3) and Glu144 (OE2) (black) and water W1 (water molecule 403 in the case of mEos $4 \mathrm{~b}$ and water molecule 2032 in the case of IrisFP) (red) during the six MD runs of mEos4b (left) and IrisFP chain C (right). The insets show a zoom of the 3.6-4.6 ns interval. (C) Time evolution of the distance between the Glu212 (OE1) carboxyl oxygen and the chromophore imidazolinone nitrogen (N15) during the six concatenated MD runs of mEos4b and IrisFP chain C. Pictures on the left of each panel highlight the studied parameters.

trans state is also stabilized by the ability of Ser142, which establishes a strong $\mathrm{H}$-bond with the hydroxybenzylidene moiety in the cis state, to orient toward the surrounding solvent and find a compensating $\mathrm{H}$-bonding partner in Glu158 once the chromophore has been isomerized (Figure 1D). ${ }^{38}$ Protonation of the chromophore in the trans state is then explained by the change in electrostatic environment relative to the cis state. ${ }^{38,48,52}$

In IrisFP, the Phe173Ser single-point mutation induces a reorientation of Met159 that creates a pocket above the chromophore hydroxybenzylidene ring and greatly facilitates chromophore isomerization. Interestingly, the absence of such a pocket in $\mathrm{mEos} 4 \mathrm{~b}$ does not preclude such isomerization. We therefore investigated further how this PCFP still manages to sustain the chromophore in the trans configuration.

First, we noticed that, in comparison to IrisFP, the distance between Glu144 and the hydroxyl group of the chromophore hydroxybenzylidene moiety is lengthened ( $3.5 \AA$ vs $2.9 \AA$ ), and the only strong hydrogen bond formed by this group involves a water molecule (WAT403, $2.5 \AA$ ), which is tightly held in place by Gly155, Glu144, and Thr175. Thus, the interaction between the chromophore and Glu144 is weakened. Furthermore, in contrast to IrisFP, the conserved Glu212 does not establish H-bonding to the imidazolinone moiety of the chromophore (Figure 1D). Finally, the chromophore in dark mEos $4 \mathrm{~b}$ seems to adopt higher tilt and twist dihedral and methylene bridge bond angles $\left(-25^{\circ}, 52^{\circ}\right.$, and $130^{\circ}$, respectively), as compared to $\operatorname{IrisFP}\left(-4^{\circ}, 40^{\circ}\right.$, and $125^{\circ}$, respectively). However, we noticed that the electron density in the final $2 F_{\mathrm{o}}-F_{\mathrm{c}}$ map (Figure S8B) does not strictly overlay our best-refined model of the chromophore, which suggests the possible existence of multiple unresolved conformations. The higher temperature factor of the conjugated part of the chromophore $\left(40.7 \AA^{2}\right)$ as compared to the average $\left(32.8 \AA^{2}\right.$ ) in this model agrees with this hypothesis. Ensemble refinement further supported this hypothesis, which indicates that, whereas the bright-state structure is characterized by stable amino acid conformations, a much wider variety of conformations is found in the dark-state chromophore and its environment (Figure 1E,F). Consequently, mEos4b shows a remarkably higher variation in the twist dihedral than that observed in IrisFP (Figure S11 and Table S2), indicative of mEos $4 b$ 's chromophore exploration for alternative interaction partners.

Molecular Dynamics Simulations. To explore the mechanistic basis for such a dynamic chromophore in the Green $_{\text {off }}$ state, we carried out MD simulations and compared the results to those obtained on IrisFP. As IrisFP is a tetrameric FP, we studied all four chains, each with the two different Ser142 conformations observed in the crystal 
structure (see materials and methods in the Supporting Information for a description of the differences between IrisFP monomers). ${ }^{38}$ In the following, we report on the average of those eight simulations, but in Figure 2 we show only the results corresponding to the most populated IrisFP conformation (chain C with the Ser142 inward orientation; results on all other conformations can be found in Figure S12, Figure S13, Table S3, and supplementary note). Strikingly, in $\mathrm{mEos} 4 \mathrm{~b}$, a large variation in tilt and twist dihedral angles of the off-switched chromophore is observed, with transitions between two main substates (Figure 2A). These transitions are not seen in IrisFP, suggestive of a more flexible chromophore in $\mathrm{mEos} 4 \mathrm{~b}$, with a loose attachment to the surrounding amino acids and water molecules. Of note, the average values of the tilt and twist angles derived from the MD simulations of $\mathrm{mEos} 4 \mathrm{~b}$ are lower than those extracted from the refined X-ray structure of the chromophore (Table S3). We tentatively explain this finding by the fact that the latter structure likely represents a superposition of several more planar conformations. In fact, the MD simulations show that the mEos $4 \mathrm{~b}$ chromophore rapidly toggles between two main conformations exhibiting different $\mathrm{H}$-bonding patterns (Figure 2B). In the first conformation (62\% of the time, Tables S3 and S4), the hydroxybenzylidene oxygen (O3, see Figure S14 for chromophore atom names) maintains a hydrogen bond with Glu144, whereas this bond is broken and replaced with a hydrogen bond to a water molecule (here called W1) in the second conformation. Thus, $\mathrm{O} 3$ alternately donates its proton to Glu144 or W1. In the case of IrisFP, similar MD simulations showed a highly predominant conformation (observed $90 \%$ of the time, Table S4) where O3 donates its proton to Glu144. In mEos $4 \mathrm{~b}$, the transitions between the two main chromophore conformations occur over periods of a few nanoseconds. However, zooming on a $1 \mathrm{~ns}$ time interval (Figure 2B, insets) reveals that hydrogen bond breaking happens also on smaller time scales, from a few picoseconds to a few 100 ps. Interestingly, the H-bonding conformational switch at the level of the hydroxybenzylidene oxygen correlates with the changes in tilt and twist dihedral angles, as is apparent from Figure 2A,B (Pearson coefficients between the time-dependent Glu144(OE2)-CRO(O3) distance and chromophore tilt and twist dihedrals are -0.44 and 0.32 , respectively).

From the hydrogen-bonding triad Glu144-Arg66-Glu212 observed in the crystal structure (Figure S10), the Glu144Arg66 H-bond is continuously established in all simulations for both mEos $4 \mathrm{~b}$ and IrisFP. However, this is not the case for the Arg66-Glu212 bond. In mEos4b, the Arg66(NH2)-Glu212(OE2) interaction exists only $41 \%$ of the time, while in IrisFP it is almost always broken and a water molecule mediates the interaction between the two partners all along the simulations. In IrisFP, Arg66(NH2) establishes hydrogen bonding with His194, which introduces a difference in the bonding pattern around the chromophore's imidazolinone moiety between the two proteins. In IrisFP, the N15 atom of the chromophore interacts with Glu212 during $86 \%$ of the simulation time (Figure 2C and Table S4). Furthermore, the imidazolinone oxygen $(\mathrm{O} 25)$ remains $\mathrm{H}$-bonded to a water molecule during $43 \%$ of the time, in addition to the stable H-bond to Arg91. In contrast, in mEos $4 \mathrm{~b}$, the imidazolinone moiety establishes no other hydrogen bonds than the one with Arg91.

Overall, the MD simulations clearly suggest a less stable offstate chromophore in mEos $4 b$ as compared to IrisFP, in agreement with the results of our ensemble refinements based on crystallographic data. The dynamic nature of the chromophore in mEos $4 \mathrm{~b}$ in the off-switched state can be linked to the reduced number of hydrogen bonds established with neighboring residues of the chromophore pocket. The average number of hydrogen bonds established by the hydroxybenzylidene oxygen, the imidazolinone oxygen, and the imidazolinone nitrogen with a free electron pair $(\mathrm{O} 3, \mathrm{O} 25$, and N15 atoms, respectively, Figure S14) over the course of our MD simulations is 2.5 for mEos $4 b$, whereas it is 3.5 for IrisFP. By comparison, the numbers of $\mathrm{H}$-bonds extracted from the static crystallographic structures are 3.6 and 6.6, respectively (see materials and methods in the Supporting Information for a description of the H-bond calculation). These higher numbers, in particular, highlight the transient nature of the $\mathrm{H}$-bonding interactions, which cannot be accounted for in the X-ray structures. The weak anchoring of the chromophore in mEos $4 \mathrm{~b}$ could facilitate back switching to the on-state upon light excitation and hence account for the relatively high back switching quantum yield and the low switching contrast of this PCFP (Table S1).

Comparison with the mEos4b Red Long-Lived Dark State. In the red-state of mEos $4 b$, we recently observed a similar photoswitching behavior involving chromophore isomerization. ${ }^{25}$ In the corresponding off-state, named $\operatorname{Red}_{\text {off }}$ a so-called "frustrated" chromophore was found, which, based on crystallographic data, appeared even more disordered than in the Green ${ }_{\text {off }}$ state. As for Green $n_{\text {off }}$ the refined X-ray structure of $\operatorname{Red}_{\text {off }}$ should be regarded as a superposition of several subconformations. An overlay of the structures of the two dark states is shown in Figure 3. It is interesting to realize that the differences between the two structures only result from backbone breakage at Phe61 and extended electron conjugation induced by photoconversion, while the chromophore

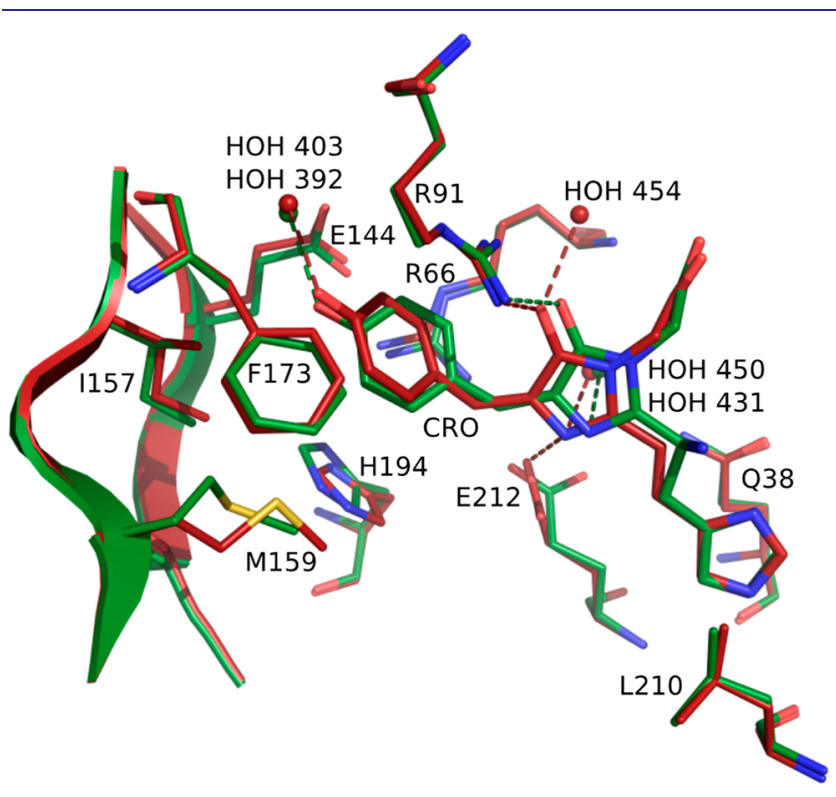

Figure 3. Comparison of the dark states in green and red mEos $4 \mathrm{~b}$. Superposition of the structures of the chromophore and its surrounding environment in the Green off $_{\text {and }}$ Red $d_{\text {off }}$ dark states of $\mathrm{mEos} 4 \mathrm{~b}$ (green and red color, respectively, PDB ID for the red longlived dark state: 6GP1). H-bonds possibly established between the conjugated part of the chromophore and the surrounding pocket are indicated by dashed lines (H-bond criterium: distance smaller than or equal to $3.2 \AA$ ). 
environment in green and red mEos $4 \mathrm{~b}$ remains essentially unchanged. Interestingly, in $\operatorname{Red}_{\text {off }}$ the distance between the phenol group of the chromophore and Glu144 is now $>4.2 \AA$, strongly suggesting that the chromophore loses completely the interaction with Glu144 that is seen transiently in Green off $_{\text {and }}$ and almost at all times in IrisFP. Instead, the chromophore only has the possibility to make a hydrogen bond with a water molecule. However, relatively robust $\mathrm{H}$-bonding is maintained in $\operatorname{Red}_{\text {off }}$ at the level of the imidazolinone moiety (five possible H-bonds versus six in the red-on state). Therefore, the highly dynamic nature of the dark state in red mEos $4 \mathrm{~b}$ may not only relate to the $\mathrm{H}$-bonding pattern linking the chromophore to the $\beta$-barrel. Here, it is important to keep in mind that, as compared to Green $_{\text {off }}$, backbone breakage and extended conjugation in $\operatorname{Red}_{\text {off }}$ are expected to also result in significant changes in electrostatic interactions maintained by the chromophore. This is, for example, reflected in the fact that the $\pi$-cation interaction between Arg66 and the chromophore, seen in Green off, is prevented in Red $_{o f f}$, this role being taken by the better positioned Arg91 (Figure S15). In addition to $\mathrm{H}$ bonding, electrostatic interactions also contribute to chromophore stability and largely dictate switching capability, as was recently demonstrated in Dronpa2. ${ }^{53}$ Overall, we speculate that the dynamic nature of the chromophore in the red-off state of mEos $4 b$ derives from a combination of weak H-bonding and electrostatic interactions, notably at the level of the hydroxybenzylidene moiety. This may be related to the difficulty encountered to date to engineer red RSFPs displaying a high switching contrast. ${ }^{54}$

Raman Spectroscopy. To evaluate how irreversible photoconversion and reversible photoswitching may modify mEos4b structural dynamics in the ground state, we collected Raman spectra in its green and red forms, as well as in the corresponding dark states. Using flash-cooled mEos $4 \mathrm{~b}$ crystals and laser excitation at $785 \mathrm{~nm}$ in a preresonance mode, ${ }^{55}$ we obtained high-resolution and high signal-to-noise ratio spectra, notably thanks to the highly concentrated crystalline samples and the narrower bands at low temperature. ${ }^{56}$ The Raman spectrum of green mEos $4 \mathrm{~b}$ (Figures $4 \mathrm{~A}$ and S16) displays bands similar to those previously observed on green IrisFP $^{50}$ and FP chromophores, ${ }^{57}$ which allows one to propose partial band assignment (Table S5). The spectrum is also globally similar to that obtained on EYQ1, a green-yellow hydrozoan RSFP engineered from GFP, ${ }^{58}$ although substantially more bands can be observed in the case of mEos $4 \mathrm{~b}$. The 1500-1650 $\mathrm{cm}^{-1}$ window is of particular interest, with strong bands associated with delocalized modes involving the chromophore imidazolinone moiety and exocyclic double bond and known to be highly sensitive to the protonation and conjugation state of the chromophore. ${ }^{23}$ Upon photoconversion, the Raman spectrum of red mEos $4 b$, although globally similar to that of green mEos $4 \mathrm{~b}$, revealed several specific changes (Figure $4 \mathrm{~A}$ and Table S5). Most prominent was the appearance of a strong band at $1638 \mathrm{~cm}^{-1}$ and the significant shifts of bands at 1540 , $1286,1173,935$, and $566 \mathrm{~cm}^{-1}$ (green form) to 1529,1295 , 1179,940 , and $570 \mathrm{~cm}^{-1}$ (red form). Of note, although some bands appear conserved (supplementary note), this spectrum significantly differs from those of eqFP611 and dsRed, the only two red fluorescent proteins, to our knowledge, previously investigated by Raman spectroscopy. ${ }^{59,60}$ Recently, however, femtosecond stimulated Raman spectra of LEA, an engineered ancestral photoconvertible fluorescent protein, were also published. ${ }^{32} \mathrm{~A}$ number of Raman bands appear common
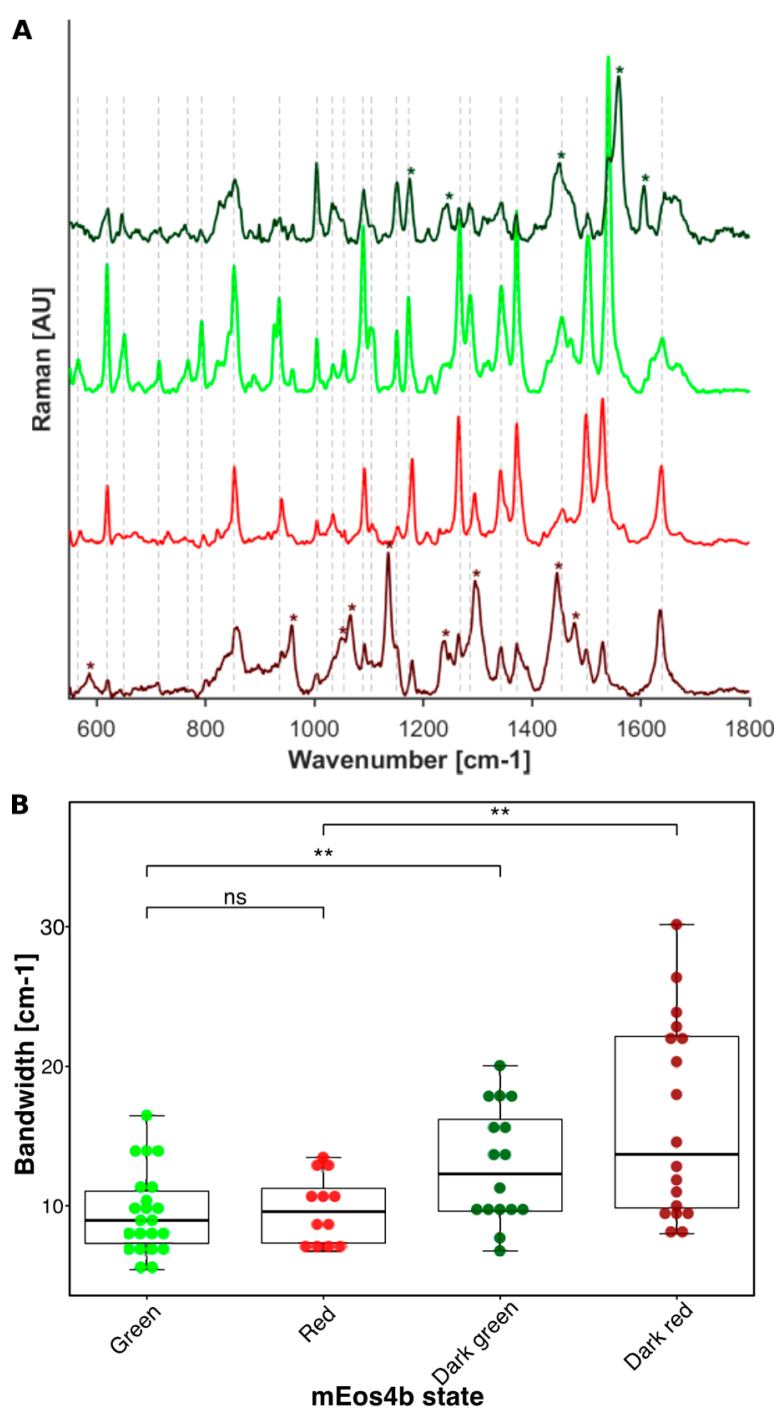

Figure 4. Raman signature of mEos $4 b$. (A) Raman spectra of mEos $4 b$ in the green bright state (green), green off-state (dark green), photoconverted red state (red), and red off-state (dark red) measured at $\mathrm{pH} 7.4$ on flash frozen crystals. Red-state crystals were obtained after photoconversion of green crystals by sunlight. Vertical dashed lines are shown at the positions of the main Raman bands in the green bright state to guide the eye. Asterisks denote the positions of specific bands associated with the green and red off-states. (B) Swarm and box plots of the width of Raman bands for the four mEos4b states. **: $P$ < $10^{-2}, t$ test; the thick black line, box plot, and whiskers show, respectively, the mean, 25-75 quartiles, and adjacent points.

between green mEos $4 \mathrm{~b}$ and green LEA, but a striking difference is the much less pronounced bands observed for LEA in the $1400-1550 \mathrm{~cm}^{-1}$ window. Upon photoconversion, the appearance of bands at 937, 1177, 1294, 1503, and 1531 $\mathrm{cm}^{-1}$ in LEA correlates well with observations made in red mEos $4 \mathrm{~b}$. Interestingly, although using preresonance excitation at $785 \mathrm{~nm}$ prevents us from specifically exciting the green chromophore after photoconversion, our spectra do not suggest the UV-induced buildup of a dead-end species with a characteristic Raman shift of $1340 \mathrm{~cm}^{-1}$, as was observed in LEA. In contrast, in the case of mEos $4 b$, inspection of the significantly shifted bands between the green and the red states rather indicates that the Raman spectrum of red mEos $4 b$ is essentially free of significant contribution from the remaining green mEos $4 \mathrm{~b}$. To reconcile this observation with the only 
A

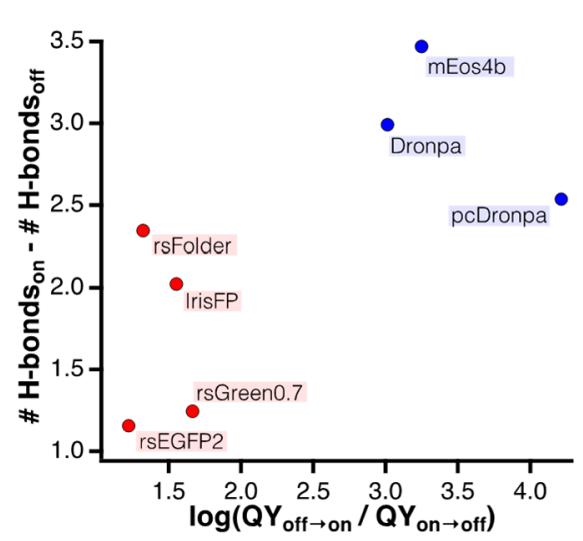

B

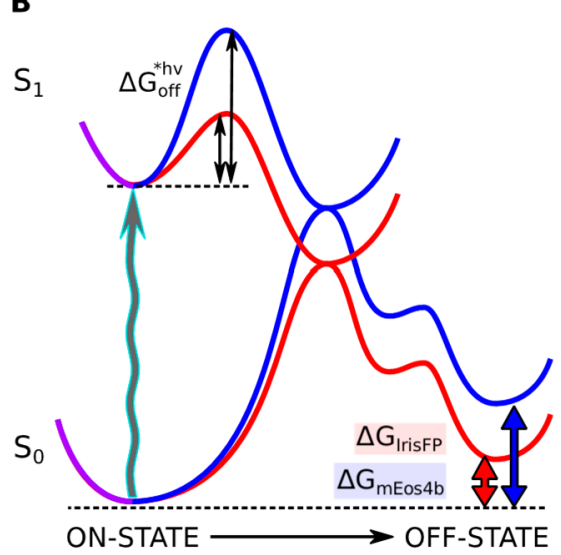

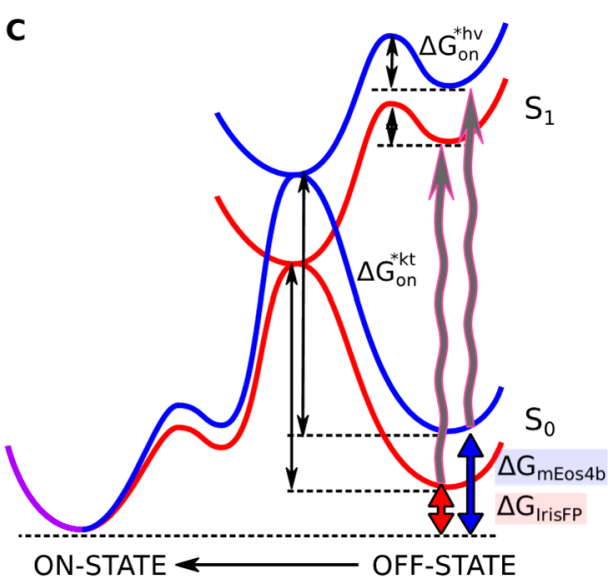

Figure 5. Correlation between relative chromophore H-bonding in the on- and off-states of RSFPs and ratio of photoswitching quantum yields. (A) Plot of the difference in the number of possible H-bonds linking the chromophore and the surrounding protein matrix seen in crystallographic structures of green RSFPs in their on- and off-states versus the ratio of measured photoswitching quantum yields (Pearson correlation coefficient $=$ 0.69). Used PDB IDs for the on- and off-states, respectively, are 2IE2, 2POX (Dronpa), 4HQ8, 4HQ9 (pcDronpa), 2VVH, 2VVI (IrisFP), 6GOY, 6GOZ (mEos4b), 4XOW, 4XOV (rsGreen0.7), 5DTX, 5DTY (rsEFGP2), and 5DTZ, 5DU0 (rsFolder). On-off and off-on photoswitching quantum yields were extracted from Moeyaert et al., ${ }^{41}$ Adam et al., ${ }^{38} \mathrm{El}$ Khatib et al., ${ }^{45}$ and Duwé et al., ${ }^{10}$ except those of mEos4b, which were determined in the present work (Table S1). Blue and red colors refer to arrow colors in panels B and C. (B and C) Tentative schemes accounting for the larger ratio $Q Y_{\text {off } \rightarrow \text { on }} / Q Y_{\text {on } \rightarrow \text { off }}$ in mEos4b (blue) as compared to IrisFP (red), with (B) representing on-off switching and (C) representing off-on switching. The similar absorption wavelengths in the on- and off-states of mEos $4 \mathrm{~b}$ and IrisFP allow one to shift the energy diagram of one of the two proteins to superpose their $S_{0}$ and $S_{1}$ energy levels in the on-state. Energy barriers for isomerization are higher in mEos $4 \mathrm{~b}$ than in IrisFP, both in the ground and in the excited states (hence the lower on-off and off-on switching quantum yields, as well as slower thermal recovery for $\mathrm{mEos} 4 \mathrm{~b}$ ). Loose binding of the mEos $4 \mathrm{~b}$ chromophore in the off-state, in particular due to weak $\mathrm{H}$-bonding, raises the free energy of conformations prone to back photoswitching. Blue (red) double-sided arrows refer to free-energy differences between the on and off ground states of $\mathrm{mEos} 4 \mathrm{~b}$ (IrisFP). Wavy arrows denote light excitation with cyan and violet light. Additional energy barriers in the ground state represent protonation (deprotonation) after cis-trans (trans-cis) isomerization. Energies not drawn to scale.

partial photoconversion of PCFPs reported in single-molecule studies, $^{33,34}$ we propose the following interpretation: the missing fraction of red chromophores could be attributed either to nonmature or to photobleached chromophores, both of which would not be seen in the Raman spectra because of a lack of response to preresonance excitation at $785 \mathrm{~nm}$. Overall, our data suggest that the conclusion drawn from LEA to account for incomplete photoconversion in PCFPs may not fully apply to the case of mEos variants.

The Raman spectra of the mEos $4 b$ long-lived dark states, Green $_{\text {off }}$ and $\operatorname{Red}_{\text {off }}$, although containing a residual contribution from molecules in the corresponding fluorescent states (see materials and methods in the Supporting Information), drastically differ from those of the fully occupied fluorescent states (Figure 4), notably with pronounced changes in the $1500-1650 \mathrm{~cm}^{-1}$ window. We also compared these spectra with spectra collected under low-pH conditions, in the absence of illumination (Figure S16, Figure S17, and Table S5). For the Green $_{\text {off }}$ state, changes are consistent with the signature of a protonated chromophore, ${ }^{38,61}$ as expected from spectroscopic data (Figure 1A). However, slight band shifts between the dark and low-pH spectra (Figure S17) highlight the different isomerization states of the chromophore and/or the different protonation states of chromophore-interacting residues. Similar shifts were noticed in the case of EYQ1. ${ }^{58}$ For the red state, the changes observed upon off-switching also point toward a protonated chromophore, although in this case the differences between the low $\mathrm{pH}$ and the dark Raman spectra are more pronounced. Notably, bands at 1564 and $1602 \mathrm{~cm}^{-1}$ are not seen in the dark spectrum, and all bands in the 1500$1600 \mathrm{~cm}^{-1}$ region display reduced intensities, consistent with the notion of a highly distorted chromophore. ${ }^{25}$
Strikingly, the Raman spectra of Green off and Red off show bands of significantly increased width (Figure 4B, Figure S16, and see materials and methods in the Supporting Information for details on bandwidth analysis). We tentatively assign this increase to the highly dynamic chromophores in the dark states, that result from the several visited conformations, ${ }^{62}$ in line with our results from X-ray crystallography and molecular dynamics simulations. Of note, we find that the low-pH Raman spectra of green and red mEos $4 b$ also exhibit increased bandwidths, although to a lower extent (Figure S18). This suggests that protonation of the chromophore by either illumination or $\mathrm{pH}$ lowering is the main cause of destabilization.

\section{DISCUSSION AND CONCLUSION}

Our work provides an extended picture of the complex photophysics of a PCFP and highlights the pronounced biphotochromic character of the mEos variants due to frustrated chromophore cis-trans isomerization and protonation. The data reveal the subtle influence that the color state of the chromophore can have on reversible photoswitching and points at the highly dynamic character of the photoswitched states in both green and red mEos $4 \mathrm{~b}$. Several studies, notably based on NMR spectroscopy, ${ }^{63,64}$ recently pointed at the dynamical behavior of RSFPs in their off-states. This dynamic behavior mostly results from the reduced capability of protonated chromophores to maintain interactions with the surrounding $\beta$-barrel. Our work, however, suggests that the magnitude of this dynamic behavior depends on the fluorescent protein under consideration, being much more pronounced in mEos $4 \mathrm{~b}$ than in IrisFP. An intrinsic property of FP chromophores is to undergo cis-trans isomerization upon 
light excitation. Despite the stiffness of the FP scaffold, we propose that such isomerization can never be completely prevented and ends up in trans chromophores that may be illaccommodated ("frustrated"), while at the same time being trapped due to high free energy barriers between the cis and trans states (e.g., caused by steric hindrance during isomerization).

We speculate that one of the most important factors controlling switching contrast in green RSFPs is the relative stability of the cis and trans states, notably via the number of $\mathrm{H}$-bonds maintained by the chromophore in each state. This speculation is in line with ultrafast spectroscopic experiments highlighting how $\mathrm{H}$-bonding interactions control chromophore isomerization quantum yield in bacteriophytochromes. ${ }^{65,66}$ Quantum mechanics/molecular dynamics simulations performed on Dronpa also revealed favored on-off photoswitching for chromophore configurations characterized by a low number of $\mathrm{H}$-bonding interactions. ${ }^{67}$ Although other factors may come into play (see below), the switching contrast (C) in RSFPs is primarily dictated by the on-off and off-on switching quantum yields ( $\left.Q Y_{\text {on } \rightarrow \text { off }}, Q Y_{\text {off } \rightarrow \text { on }}\right)$ and the extinction coefficients of both the on- and the off-states at the actinic wavelength $\left(\varepsilon_{488, \text { on }}, \varepsilon_{488 \text {, off }}\right)$, and it can be approximated as follows:

$$
C=\frac{\varepsilon_{488, \mathrm{on}} \times Q Y_{\text {on } \rightarrow \text { off }}}{\varepsilon_{488, \mathrm{off}} \times Q Y_{\mathrm{off} \rightarrow \mathrm{on}}}
$$

As switching quantum yields relate to microscopic switching rates in $S_{1}$ electronically excited states, their logarithm is expected to scale with the Gibbs free energy difference $\Delta G^{*}$ between $S_{1}$ and the transition state to be crossed along the switching pathway. If $\mathrm{H}$-bonding contributes significantly to $\Delta G^{*}$ by raising or lowering the free energy of $S_{0}$ and $S_{1}$ in both the on- and the off-states of an RSFP, a correlation should be observed between $\log \left(Q Y_{\text {off } \rightarrow \text { on }} / Q Y_{\text {on } \rightarrow \text { off }}\right)$ and $\Delta N_{\mathrm{H}}$, the difference in the number of $\mathrm{H}$-bonds maintained by the chromophore in the on- and off-states. Such a plot is shown in Figure 5A, which gathered data on all PCFPs and RSFPs for which both the structures of the green on- and off-states and the corresponding switching quantum yields have been measured. The plot indeed reveals a significant correlation (Pearson correlation of $0.69, P<0.05$ ). This result should however be considered only qualitatively, when one considers the factors listed below: first, photoswitching is a complex photophysical process not characterized by a single transition barrier for both the on-off and the off-on switching processes, and thus the height of the on-off barrier relative to that of the off-on barrier will also contribute; second, the number of $\mathrm{H}$-bonds measured in static crystallographic structures depends on the choice of an arbitrary distance threshold (see materials and methods in the Supporting Information) and does not account for transient interactions, as revealed in the present study by MD simulations; third, switching quantum yields extracted from the literature, as done here, have been measured under a variety of illumination and environmental conditions, which likely introduced substantial variability; fourth, electrostatic interactions have been shown to also play a key role in controlling switching quantum yields and likely contribute to the activation free energy difference between the on-off and off-on switching pathways; ${ }^{53}$ fifth, our H-bond inventory of the ground-state structures may not fully reflect the $\mathrm{H}$-bonding patterns in the excited state in which photoswitching takes place; and sixth, the influence of the crystal lattice on the conformation and dynamics of the chromophore, and therefore on our H-bond inventory, could be non-negligible, as was recently demonstrated in the case of rsEGFP2. ${ }^{6}$ These limitations likely account for the lack of a clear correlation observed in Figure 5A within the red and blue RSFPs subgroups. Yet, the fact that an overall correlation is observed despite the crude simplifications made suggests that $\mathrm{H}$-bonding is indeed a critical factor controlling switching quantum yields in RSFPs. This finding is after all not surprising in view of the fact that a single $\mathrm{H}$-bond is typically worth an energy of several $k T$ and may thus affect switching rates by orders of magnitude. Tentative energy schemes are shown in Figure $5 \mathrm{~B}$ and $\mathrm{C}$, which illustrate the proposed scenario. These schemes represent the reaction coordinates during on-off switching (Figure 5B) and off-on switching (Figure 5C), with ground-state protonation after cis-trans isomerization and ground-state deprotonation after trans-cis isomerization, ${ }^{69}$ but we do not exclude a more complex switching mechanism with more transition barriers. ${ }^{70}$

The transition barriers between the on- and off-states are higher in mEos $4 b$ as compared to IrisFP in both directions, in line with the smaller switching quantum yields and slower thermal recovery in mEos $4 b$ (Table S1 and Figure S3). These higher barriers are likely a consequence of a stronger steric hindrance during isomerization in mEos $4 \mathrm{~b}$ as compared to IrisFP, linked to the Phe173Ser mutation in the latter FP. Yet, the rate of off-on switching relative to that of on-off switching is much higher in mEos $4 b$ because of the raised free energy of the off-state relative to that of the on-state. This is mainly a consequence of the lower number of $\mathrm{H}$-bonding interactions in the $\mathrm{mEos} 4 \mathrm{~b}$ off-state as compared to IrisFP. The energy barrier $\left(\Delta G^{*}\right)$ to be crossed during off-on photoswitching is thus reduced relative to that during on-off photoswitching, leading to an increased ratio $Q Y_{\text {off } \rightarrow \text { on }} / Q Y_{\text {on } \rightarrow \text { off }}$.

Interestingly, we noticed that the red and blue subgroups in Figure 5A gather faster-switching and slower-switching RSFPs, respectively. This finding additionally suggests that a correlation might exist between switching efficiency and relative chromophore $\mathrm{H}$-bonding in the on and off ground states of RSFPs. The reason behind this correlation is presently unclear and will need to be investigated in future studies.

Although the switching yield ratio $Q Y_{\text {off } \rightarrow \text { on }} / Q Y_{\text {on } \rightarrow \text { off }}$ plays a primary role, the switching contrast in RSFPs is also dictated by the sensitivity to light excitation of both the on- and the offstates at the actinic wavelength (ratio $\varepsilon_{488 \text {,on }} / \varepsilon_{488 \text {,off }}$ ). Therefore, the correlation between experimentally measured switching contrast and $\Delta N_{\mathrm{H}}$ is less pronounced (Figure S19). Furthermore, the effective switching contrast ultimately controlling image quality in nanoscopy approaches depends on yet other factors, such as a possible residual fluorescence of the off-state or a contribution from the short-lived dark state pinpointed in this work or from other weakly populated dark states still to be identified. The switching contrast also typically varies along photofatigue decay, possibly due to multiple subpopulations of RSFPs experiencing different photophysical behaviors. $^{27}$ Of note, crystallographic structures of RSFPs in their off-state typically relate to the first switching cycle, as done here, and it will be interesting in the future to evaluate how these structures might subtly differ from those collected after multiple switching. Further work is thus needed to better 
understand the mechanisms fine-tuning the switching contrast in RSFPs.

In conclusion, our work allowed us to decipher the main switching mechanism at play in green mEos $4 \mathrm{~b}$. The critical importance of $\mathrm{H}$-bonding patterns between the chromophore and the surrounding protein matrix in controlling photoswitching, as proposed here, adds to the recently demonstrated role of electrostatic interactions. ${ }^{53}$ The dynamic behavior of the chromophore, driven by both types of interactions, will be critical to consider for the future rational engineering of novel PTFPs.

\section{ASSOCIATED CONTENT}

\section{SI Supporting Information}

The Supporting Information is available free of charge at https://pubs.acs.org/doi/10.1021/jacs.0c01880.

Materials and methods, supplementary notes on molecular dynamics simulations and Raman data, supplementary figures, and supplementary tables (PDF)

\section{AUTHOR INFORMATION}

\section{Corresponding Author}

Dominique Bourgeois - Institut de Biologie Structurale, Universite Grenoble Alpes, CEA, CNRS, Grenoble 38044, France; (1) orcid.org/0000-0002-1862-7712; Email: dominique.bourgeois@ibs.fr

\section{Authors}

Elke De Zitter - Department of Chemistry, KU Leuven, Heverlee 3001, Belgium

Jacqueline Ridard - Institut de Chimie Physique UMR8000, Universite Paris-Saclay, CNRS, Orsay 91405, France

Daniel Thédié - Institut de Biologie Structurale, Université Grenoble Alpes, CEA, CNRS, Grenoble 38044, France

Virgile Adam - Institut de Biologie Structurale, Université Grenoble Alpes, CEA, CNRS, Grenoble 38044, France; (ㄱ) orcid.org/0000-0003-2209-7846

Bernard Lévy - Institut de Chimie Physique UMR8000, Universite Paris-Saclay, CNRS, Orsay 91405, France

Martin Byrdin - Institut de Biologie Structurale, Université Grenoble Alpes, CEA, CNRS, Grenoble 38044, France

Guillaume Gotthard - Structural Biology Group, European Synchrotron Radiation Facility, Grenoble 38000, France; (1) orcid.org/0000-0003-2830-0286

Luc Van Meervelt - Department of Chemistry, KU Leuven, Heverlee 3001, Belgium; - orcid.org/0000-0003-2186-5209

Peter Dedecker - Department of Chemistry, KU Leuven, Heverlee 3001, Belgium

Isabelle Demachy - Institut de Chimie Physique UMR8000, Universite Paris-Saclay, CNRS, Orsay 91405, France

Complete contact information is available at:

https://pubs.acs.org/10.1021/jacs.0c01880

\section{Author Contributions}

${ }^{\perp}$ J.R. and D.T. contributed equally.

\section{Notes}

The authors declare no competing financial interest.

\section{ACKNOWLEDGMENTS}

This work was supported by the Agence Nationale de la Recherche (grant no. ANR-17-CE11-0047-01 to D.B.) and used the Cryobench and M4D imaging platform of the Grenoble Instruct-ERIC Center (ISBG: UMS 3518 CNRSCEA-UGA-EMBL) with support from FRISBI (grant no. ANR-10-INBS-05-02) and GRAL, a project of the Universite Grenoble Alpes graduate school (Ecoles Universitaires de Recherche) CBH-EUR-GS (ANR-17-EURE-0003) within the Grenoble Partnership for Structural Biology (PSB). We thank the staff of beamline ID-23-1 from the ESRF, Grenoble, France. Molecular dynamics simulations were carried out using the computing facilities of the Institut de Chimie Physique. We thank A. Royant and P. Carpentier for stimulating discussions on Raman spectroscopy. E.D.Z. thanks the Research Foundation Flanders (FWO) for a doctoral fellowship and travel bursary.

\section{REFERENCES}

(1) Adam, V.; Berardozzi, R.; Byrdin, M.; Bourgeois, D. Phototransformable Fluorescent Proteins: Future Challenges. Curr. Opin. Chem. Biol. 2014, 20, 92-102.

(2) Mizuno, H.; Mal, T. K.; Tong, K. I.; Ando, R.; Furuta, T.; Ikura, M.; Miyawaki, A. Photo-Induced Peptide Cleavage in the Green-toRed Conversion of a Fluorescent Protein. Mol. Cell 2003, 12 (4), $1051-1058$.

(3) Betzig, E.; Patterson, G. H.; Sougrat, R.; Lindwasser, O. W.; Olenych, S.; Bonifacino, J. S.; Davidson, M. W.; Lippincott-Schwartz, J.; Hess, H. F. Imaging Intracellular Fluorescent Proteins at Nanometer Resolution. Science 2006, 313 (5793), 1642-1645.

(4) Shen, H.; Landes, C. F.; Shuang, B.; Tauzin, L. J.; Wang, W.; Baiyasi, R; Moringo, N. Single Particle Tracking: From Theory to Biophysical Applications. Chem. Rev. 2017, 117, 7331-7376.

(5) Nemet, I.; Ropelewski, P.; Imanishi, Y. Applications of Phototransformable Fluorescent Proteins for Tracking the Dynamics of Cellular Components. Photochemical and Photobiological Sciences. 2015, 14, 1787-1806.

(6) Bourgeois, D.; Adam, V. Reversible Photoswitching in Fluorescent Proteins: A Mechanistic View. IUBMB Life 2012, 64 (6), 482-491.

(7) Grotjohann, T.; Testa, I.; Reuss, M.; Brakemann, T.; Eggeling, C.; Hell, S. W.; Jakobs, S. RsEGFP2 Enables Fast RESOLFT Nanoscopy of Living Cells. eLife 2012, 2012 (1), 1-14.

(8) Zhang, X.; Zhang, M.; Li, D.; He, W.; Peng, J.; Betzig, E.; Xu, P. Highly Photostable, Reversibly Photoswitchable Fluorescent Protein with High Contrast Ratio for Live-Cell Superresolution Microscopy. Proc. Natl. Acad. Sci. U. S. A. 2016, 113 (37), 10364-10369.

(9) Zhang, X.; Chen, X.; Zeng, Z.; Zhang, M.; Sun, Y.; Xi, P.; Peng, J.; Xu, P. Development of a Reversibly Switchable Fluorescent Protein for Super-Resolution Optical Fluctuation Imaging (SOFI). ACS Nano 2015, 9 (3), 2659-2667.

(10) Duwé, S.; De Zitter, E.; Gielen, V.; Moeyaert, B.; Vandenberg, W.; Grotjohann, T.; Clays, K.; Jakobs, S.; Van Meervelt, L.; Dedecker, P. Expression-Enhanced Fluorescent Proteins Based on Enhanced Green Fluorescent Protein (EGFP) for Super-Resolution Microscopy. ACS Nano 2015, 9 (10), 9528-9541.

(11) Rosenbloom, A. B.; Lee, S. H.; To, M.; Lee, A.; Shin, J. Y.; Bustamante, C. Optimized Two-Color Super Resolution Imaging of Drp1 during Mitochondrial Fission with a Slow-Switching Dronpa Variant. Proc. Natl. Acad. Sci. U. S. A. 2014, 111 (36), 13093-13098.

(12) Alieva, N. O.; Konzen, K. A.; Field, S. F.; Meleshkevitch, E. A.; Hunt, M. E.; Beltran-Ramirez, V.; Miller, D. J.; Wiedenmann, J.; Salih, A.; Matz, M. V. Diversity and Evolution of Coral Fluorescent Proteins. PLoS One 2008, 3 (7), No. e2680.

(13) Grotjohann, T.; Testa, I.; Leutenegger, M.; Bock, H.; Urban, N. T.; Lavoie-Cardinal, F.; Willig, K. I.; Eggeling, C.; Jakobs, S.; Hell, S. W. Diffraction-Unlimited All-Optical Imaging and Writing with a Photochromic GFP. Nature 2011, 478 (7368), 204-208.

(14) Bizzarri, R.; Serresi, M.; Cardarelli, F.; Abbruzzetti, S.; Campanini, B.; Viappiani, C.; Beltram, F. Single Amino Acid 
Replacement Makes Aequorea Victoria Fluorescent Proteins Reversibly Photoswitchable. J. Am. Chem. Soc. 2010, 132 (1), 85-95.

(15) Ando, R.; Mizuno, H.; Miyawaki, A. Regulated Fast Nucleocytoplasmic Shuttling Observed by Reversible Protein Highlighting. Science 2004, 306 (5700), 1370-1373.

(16) Berardozzi, R.; Adam, V.; Martins, A.; Bourgeois, D. Arginine 66 Controls Dark-State Formation in Green-to-Red Photoconvertible Fluorescent Proteins. J. Am. Chem. Soc. 2016, 138 (2), 558-565.

(17) Haupts, U.; Maiti, S.; Schwille, P.; Webb, W. W. Dynamics of Fluorescence Fluctuations in Green Fluorescent Protein Observed by Fluorescence Correlation Spectroscopy. Proc. Natl. Acad. Sci. U. S. A. 1998, 95 (23), 13573-13578.

(18) Liu, Y.; Kim, H. R.; Heikal, A. A. Structural Basis of Fluorescence Fluctuation Dynamics of Green Fluorescent Proteins in Acidic Environments. J. Phys. Chem. B 2006, 110 (47), 2413824146.

(19) Schenk, A.; Ivanchenko, S.; Röcker, C.; Wiedenmann, J.; Nienhaus, G. U. Photodynamics of Red Fluorescent Proteins Studied by Fluorescence Correlation Spectroscopy. Biophys. J. 2004, 86 (1 I), 384-394.

(20) Byrdin, M.; Duan, C.; Bourgeois, D.; Brettel, K. A Long-Lived Triplet State Is the Entrance Gateway to Oxidative Photochemistry in Green Fluorescent Proteins. J. Am. Chem. Soc. 2018, 140 (8), 28972905.

(21) Acharya, A.; Bogdanov, A. M.; Grigorenko, B. L.; Bravaya, K. B.; Nemukhin, A. V.; Lukyanov, K. A.; Krylov, A. I. Photoinduced Chemistry in Fluorescent Proteins: Curse or Blessing? Chem. Rev. 2017, 117 (2), 758-795.

(22) Vegh, R. B.; Bloch, D. A.; Bommarius, A. S.; Verkhovsky, M.; Pletnev, S.; Iwaï, H.; Bochenkova, A. V.; Solntsev, K. M. Hidden Photoinduced Reactivity of the Blue Fluorescent Protein MKalama1. Phys. Chem. Chem. Phys. 2015, 17 (19), 12472-12485.

(23) Adam, V.; Carpentier, P.; Violot, S.; Lelimousin, M. L.; Darnault, C.; Nienhaus, G. U.; Bourgeois, D. Structural Basis of XRay-Induced Transient Photobleaching in a Photoactivatable Green Fluorescent Protein. J. Am. Chem. Soc. 2009, 131 (50), 18063-18065.

(24) Roy, A.; Field, M. J.; Adam, V.; Bourgeois, D. The Nature of Transient Dark States in a Photoactivatable Fluorescent Protein. J. Am. Chem. Soc. 2011, 133 (46), 18586-18589.

(25) De Zitter, E.; Thédié, D.; Mönkemöller, V.; Hugelier, S.; Beaudouin, J.; Adam, V.; Byrdin, M.; Van Meervelt, L.; Dedecker, P.; Bourgeois, D. Mechanistic Investigation of MEos4b Reveals a Strategy to Reduce Track Interruptions in SptPALM. Nat. Methods 2019, 16 (8), 707-710.

(26) Endesfelder, U.; Malkusch, S.; Flottmann, B.; Mondry, J.; Liguzinski, P.; Verveer, P. J.; Heilemann, M. Chemically Induced Photoswitching of Fluorescent Probes-A General Concept for SuperResolution Microscopy. Molecules 2011, 16 (4), 3106-3118.

(27) Roebroek, T.; Duwé, S.; Vandenberg, W.; Dedecker, P. Reduced Fluorescent Protein Switching Fatigue by Binding-Induced Emissive State Stabilization. Int. J. Mol. Sci. 2017, 18 (10), 2015.

(28) Avilov, S.; Berardozzi, R.; Gunewardene, M. S.; Adam, V.; Hess, S. T.; Bourgeois, D. In Cellulo Evaluation of Phototransformation Quantum Yields in Fluorescent Proteins Used as Markers for SingleMolecule Localization Microscopy. PLoS One 2014, 9 (6), No. e98362.

(29) Nienhaus, K.; Nienhaus, G. U. Chromophore Photophysics and Dynamics in Fluorescent Proteins of the GFP Family. J. Phys.: Condens. Matter 2016, 28 (44), 443001.

(30) Thédié, D.; Berardozzi, R.; Adam, V.; Bourgeois, D. Photoswitching of Green MEos2 by Intense $561 \mathrm{Nm}$ Light Perturbs Efficient Green-to-Red Photoconversion in Localization Microscopy. J. Phys. Chem. Lett. 2017, 8 (18), 4424-4430.

(31) Solovyev, I. D.; Gavshina, A. V.; Savitsky, A. P. Novel Phototransformable Fluorescent Protein SAASoti with Unique Photochemical Properties. Int. J. Mol. Sci. 2019, 20 (14), 3399.

(32) Krueger, T. D.; Tang, L.; Zhu, L.; Breen, I. L.; Wachter, R. M.; Fang, C. Dual Illumination Enhances Transformation of an
Engineered Green-to-Red Photoconvertible Fluorescent Protein. Angew. Chem., Int. Ed. 2020, 59 (4), 1644-1652.

(33) Durisic, N.; Laparra-Cuervo, L.; Sandoval-Álvarez, A.; Borbely, J. S.; Lakadamyali, M. Single-Molecule Evaluation of Fluorescent Protein Photoactivation Efficiency Using an in Vivo Nanotemplate. Nat. Methods 2014, 11 (2), 156-162.

(34) Wang, S.; Moffitt, J. R.; Dempsey, G. T.; Xie, X. S.; Zhuang, X. Characterization and Development of Photoactivatable Fluorescent Proteins for Single-Molecule-Based Superresolution Imaging. Proc. Natl. Acad. Sci. U. S. A. 2014, 111 (23), 8452-8457.

(35) Mohr, M. A.; Kobitski, A. Y.; Sabater, L. R.; Nienhaus, K.; Obara, C. J.; Lippincott-Schwartz, J.; Nienhaus, G. U.; Pantazis, P. Rational Engineering of Photoconvertible Fluorescent Proteins for Dual-Color Fluorescence Nanoscopy Enabled by a Triplet-State Mechanism of Primed Conversion. Angew. Chem., Int. Ed. 2017, 56 (38), 11628-11633.

(36) Virant, D.; Turkowyd, B.; Balinovic, A.; Endesfelder, U. Combining Primed Photoconversion and UV-Photoactivation for Aberration-Free, Live-Cell Compliant Multi-Color Single-Molecule Localization Microscopy Imaging. Int. J. Mol. Sci. 2017, 18.

(37) Shroff, H.; Galbraith, C. G.; Galbraith, J. A.; White, H.; Gillette, J.; Olenych, S.; Davidson, M. W.; Betzig, E. Dual-Color Superresolution Imaging of Genetically Expressed Probes within Individual Adhesion Complexes. Proc. Natl. Acad. Sci. U. S. A. 2007, 104 (51), 20308-20313.

(38) Adam, V.; Lelimousin, M.; Boehme, S.; Desfonds, G.; Nienhaus, K.; Field, M. J.; Wiedenmann, J.; McSweeney, S.; Nienhaus, G. U.; Bourgeois, D. Structural Characterization of IrisFP, an Optical Highlighter Undergoing Multiple Photo-Induced Transformations. Proc. Natl. Acad. Sci. U. S. A. 2008, 105 (47), 1834318348 .

(39) Fuchs, J.; Böhme, S.; Oswald, F.; Hedde, P. N.; Krause, M.; Wiedenmann, J.; Nienhaus, G. U. A Photoactivatable Marker Protein for Pulse-Chase Imaging with Superresolution. Nat. Methods 2010, 7 (8), 627-630.

(40) Adam, V.; Moeyaert, B.; David, C. C.; Mizuno, H.; Lelimousin, M.; Dedecker, P.; Ando, R.; Miyawaki, A.; Michiels, J.; Engelborghs, Y.; Hofkens, J. Rational Design of Photoconvertible and Biphotochromic Fluorescent Proteins for Advanced Microscopy Applications. Chem. Biol. 2011, 18 (10), 1241-1251.

(41) Moeyaert, B.; Nguyen Bich, N.; De Zitter, E.; Rocha, S.; Clays, K.; Mizuno, H.; Van Meervelt, L.; Hofkens, J.; Dedecker, P. Green-toRed Photoconvertible Dronpa Mutant for Multimodal SuperResolution Fluorescence Microscopy. ACS Nano 2014, 8 (2), 1664-1673.

(42) Chang, H.; Zhang, M.; Ji, W.; Chen, J.; Zhang, Y.; Liu, B.; Lu, J.; Zhang, J.; Xu, P.; Xu, T. A Unique Series of Reversibly Switchable Fluorescent Proteins with Beneficial Properties for Various Applications. Proc. Natl. Acad. Sci. U. S. A. 2012, 109 (12), 44554460.

(43) Wang, S.; Chen, X.; Chang, L.; Ding, M.; Xue, R.; Duan, H.; Sun, Y. GMars-T Enabling Multimodal Subdiffraction Structural and Functional Fluorescence Imaging in Live Cells. Anal. Chem. 2018, 90 (11), 6626-6634.

(44) Fu, Z.; Peng, D.; Zhang, M.; Xue, F.; Zhang, R.; He, W.; Xu, T.; $\mathrm{Xu}, \mathrm{P}$. MEosEM Withstands Osmium Staining and Epon Embedding for Super-Resolution CLEM. Nat. Methods 2020, 17 (1), 55-58.

(45) El Khatib, M.; Martins, A.; Bourgeois, D.; Colletier, J.-P.; Adam, V. Rational Design of Ultrastable and Reversibly Photoswitchable Fluorescent Proteins for Super-Resolution Imaging of the Bacterial Periplasm. Sci. Rep. 2016, 6, 18459.

(46) Pletnev, S.; Subach, F. V.; Dauter, Z.; Wlodawer, A.; Verkhusha, V. V. A Structural Basis for Reversible Photoswitching of Absorbance Spectra in Red Fluorescent Protein RsTagRFP. J. Mol. Biol. 2012, 417 (3), 144-151.

(47) Bourgeois, D. Deciphering Structural Photophysics of Fluorescent Proteins by Kinetic Crystallography. Int. J. Mol. Sci. 2017, 18, 1187. 
(48) Henderson, J. N.; Ai, H. W.; Campbell, R. E.; Remington, S. J. Structural Basis for Reversible Photobleaching of a Green Fluorescent Protein Homologue. Proc. Natl. Acad. Sci. U. S. A. 2007, 104 (16), 6672-6677.

(49) Paez-Segala, M. G.; Sun, M. G.; Shtengel, G.; Viswanathan, S.; Baird, M. A.; Macklin, J. J.; Patel, R.; Allen, J. R.; Howe, E. S.; Piszczek, G.; Hess, H. F.; Davidson, M. W.; Wang, Y.; Looger, L. L. Fixation-Resistant Photoactivatable Fluorescent Proteins for CLEM. Nat. Methods 2015, 12 (3), 215-218.

(50) Duan, C.; Adam, V.; Byrdin, M.; Ridard, J.; Kieffer-Jaquinod, S.; Morlot, C.; Arcizet, D.; Demachy, I.; Bourgeois, D. Structural Evidence for a Two-Regime Photobleaching Mechanism in a Reversibly Switchable Fluorescent Protein. J. Am. Chem. Soc. 2013, 135 (42), 15841-15850.

(51) Byrdin, M.; Bourgeois, D. The CAL(AI)2DOSCOPE: A Microspectrophotometer for Accurate Recording of Correlated Absorbance and Fluorescence Emission Spectra. Spectrosc. Eur. 2016, 28, 14-17.

(52) Andresen, M.; Stiel, A. C.; Trowitzsch, S.; Weber, G.; Eggeling, C.; Wahl, M. C.; Hell, S. W.; Jakobs, S. Structural Basis for Reversible Photoswitching in Dronpa. Proc. Natl. Acad. Sci. U. S. A. 2007, 104 (32), 13005-13009.

(53) Romei, M. G.; Lin, C. Y.; Mathews, I. I.; Boxer, S. G. Electrostatic Control of Photoisomerization Pathways in Proteins. Science 2020, 367 (6473), 76-79.

(54) Pennacchietti, F.; Serebrovskaya, E. O.; Faro, A. R.; Shemyakina, I. I.; Bozhanova, N. G.; Kotlobay, A. A.; Gurskaya, N. G.; Bodén, A.; Dreier, J.; Chudakov, D. M.; Lukyanov, K. A.; Verkhusha, V. V.; Mishin, A. S.; Testa, I. Fast Reversibly Photoswitching Red Fluorescent Proteins for Live-Cell RESOLFT Nanoscopy. Nat. Methods 2018, 15 (8), 601-604.

(55) Von Stetten, D.; Giraud, T.; Carpentier, P.; Sever, F.; Terrien, M.; Dobias, F.; Juers, D. H.; Flot, D.; Mueller-Dieckmann, C.; Leonard, G. A.; De Sanctis, D.; Royant, A. In Crystallo Optical Spectroscopy (IcOS) as a Complementary Tool on the Macromolecular Crystallography Beamlines of the ESRF. Acta Crystallogr., Sect. D: Biol. Crystallogr. 2015, 71, 15-26.

(56) Carpentier, P.; Royant, A.; Ohana, J.; Bourgeois, D. Advances in Spectroscopic Methods for Biological Crystals. 2. Raman Spectroscopy. J. Appl. Crystallogr. 2007, 40 (6), 1113-1122.

(57) He, X.; Bell, A. F.; Tonge, P. J. Synthesis and Spectroscopic Studies of Model Red Fluorescent Protein Chromophores. Org. Lett. 2002, 4 (9), 1523-1526.

(58) Luin, S.; Voliani, V.; Lanza, G.; Bizzarri, R.; Nifosí, R.; Amat, P.; Tozzini, V.; Serresi, M.; Beltram, F. Raman Study of Chromophore States in Photochromic Fluorescent Proteins. J. Am. Chem. Soc. 2009, 131 (1), 96-103.

(59) Loos, D. C.; Habuchi, S.; Flors, C.; Hotta, J. I.; Wiedenmann, J.; Nienhaus, G. U.; Hofkens, J. Photoconversion in the Red Fluorescent Protein from the Sea Anemone Entacmaea Quadricolor. Is Cis-Trans Isomerization Involved? J. Am. Chem. Soc. 2006, 128 (19), 6270-6271.

(60) Kruglik, S. G.; Subramaniam, V.; Greve, J.; Otto, C. Resonance CARS Study of the Structure of "Green" and "Red" Chromophores within the Red Fluorescent Protein DsRed. J. Am. Chem. Soc. 2002, 124 (37), 10992-10993.

(61) Bell, A. F.; Stoner-Ma, D.; Wachter, R. M.; Tonge, P. J. LightDriven Decarboxylation of Wild-Type Green Fluorescent Protein. J. Am. Chem. Soc. 2003, 125 (23), 6919-6926.

(62) Carpentier, P. Phase Transitions Study of the Ferroelectric Crystal (CH3NH3)5BI2CL11 (MAPCB); L'université de Lille, 1995.

(63) Mizuno, H.; Mal, T. K.; Wälchli, M.; Kikuchi, A.; Fukano, T.; Ando, R.; Jeyakanthan, J.; Taka, J.; Shiro, Y.; Ikura, M.; Miyawaki, A. Light-Dependent Regulation of Structural Flexibility in a Photochromic Fluorescent Protein. Proc. Natl. Acad. Sci. U. S. A. 2008, 105 (27), 9227-9232.

(64) Christou, N. E.; Ayala, I.; Giandoreggio-Barranco, K.; Byrdin, M.; Adam, V.; Bourgeois, D.; Brutscher, B. NMR Reveals Light-
Induced Changes in the Dynamics of a Photoswitchable Fluorescent Protein. Biophys. J. 2019, 117 (11), 2087-2100.

(65) Toh, K. C.; Stojković, E. A.; Van Stokkum, I. H. M.; Moffat, K.; Kennis, J. T. M. Proton-Transfer and Hydrogen-Bond Interactions Determine Fluorescence Quantum Yield and Photochemical Efficiency of Bacteriophytochrome. Proc. Natl. Acad. Sci. U. S. A. 2010, 107 (20), 9170-9175.

(66) Mathes, T.; Ravensbergen, J.; Kloz, M.; Gleichmann, T.; Gallagher, K. D.; Woitowich, N. C.; St. Peter, R.; Kovaleva, S. E.; Stojković, E. A.; Kennis, J. T. M. Femto- to Microsecond Photodynamics of an Unusual Bacteriophytochrome. J. Phys. Chem. Lett. 2015, 6 (2), 239-243.

(67) Morozov, D.; Groenhof, G. Hydrogen Bond Fluctuations Control Photochromism in a Reversibly Photo-Switchable Fluorescent Protein. Angew. Chem., Int. Ed. 2016, 55 (2), 576-578.

(68) Chang, J.; Romei, M. G.; Boxer, S. G. Structural Evidence of Photoisomerization Pathways in Fluorescent Proteins. J. Am. Chem. Soc. 2019, 141 (39), 15504-15508.

(69) Warren, M. M.; Kaucikas, M.; Fitzpatrick, A.; Champion, P.; Timothy Sage, J.; Van Thor, J. J. Ground-State Proton Transfer in the Photoswitching Reactions of the Fluorescent Protein Dronpa. Nat. Commun. 2013, 4, 1461.

(70) Laptenok, S. P.; Gil, A. A.; Hall, C. R.; Lukacs, A.; Iuliano, J. N.; Jones, G. A.; Greetham, G. M.; Donaldson, P.; Miyawaki, A.; Tonge, P. J.; Meech, S. R. Infrared Spectroscopy Reveals Multi-Step MultiTimescale Photoactivation in the Photoconvertible Protein Archetype Dronpa. Nat. Chem. 2018, 10 (8), 845-852. 